Supporting information

\title{
Interactions between Surfactants in Solution and Electrospun Protein Fibers - Effects on Release Behavior and Fiber Properties.
}

\author{
Karen Stephansen ${ }^{1,2}$, María García-Diaz ${ }^{2}$, Flemming Jessen ${ }^{1}$, Ioannis S. Chronakis ${ }^{1 *}$, Hanne M. \\ Nielsen ${ }^{2 *}$ \\ ${ }^{1}$ National Food Institute, Technical University of Denmark, Søltofts Plads 227, DK-2800 Kgs. Lyngby, \\ Denmark. \\ ${ }^{2}$ Department of Pharmacy, Faculty of Health and Medical Sciences, University of Copenhagen, \\ Universitetsparken 2, DK-2100 Copenhagen, Denmark. \\ *Corresponding author:
}

Hanne Mørck Nielsen; mail: hanne.morck@sund.ku.dk, phone: +45 3533 63 46, fax: +45 35336001 , address: Department of Pharmacy, Faculty of Health and Medical Sciences, University of Copenhagen, Universitetsparken 2, DK-2100 Copenhagen, Denmark.

Ioannis S. Chronakis; mail:ioach@food.dtu.dk, phone: +45 452527 16, address: National Food Institute, Technical University of Denmark, Søltofts Plads 227, DK-2800 Kgs. Lyngby, Denmark 


\section{S1: Preparation of FSP}

Fresh cod (Gadus morhua) was filleted and frozen at $-30^{\circ} \mathrm{C}$. The frozen fillet was defrosted, chopped into approximately $1 \times 1 \times 2 \mathrm{~mm}$ sized pieces, placed into centrifuge tubes and centrifuged for $15 \mathrm{~min}, 18,000 \mathrm{~g}, 5^{\circ} \mathrm{C}$ (4K15, Sigma Laboratory centrifuges, Osterode am Harz, Germany). The supernatant was transferred to a petri dish, frozen, freeze dried and stored at $-60^{\circ} \mathrm{C}$.

\section{S2: Preparation of FSP-Ins fibers}

FSP $(125 \mathrm{mg} / \mathrm{mL})$ and insulin $(20 \mathrm{mg} / \mathrm{mL})$ were dissolved in HFIP, added to a syringe and placed in a syringe pump (New Era Pump Systems, Farmingdale, NY, USA). A 30 G needle (Proto Advantage, Ancaster, ON, Canada) was used. The syringe pump delivered the FSP-Ins solution with a flow rate of $0.04 \mathrm{~mL} / \mathrm{min}$. Using a high voltage power supply (Gamma High Voltage Research, Ormond Beach, FL, USA), an electric field of 25 $\mathrm{kV}$ was applied between the spinneret of the syringe and a the $5 \times 5 \mathrm{~cm}$ collector plate made of stainless steel covered by alumina foil. The distance between the syringe tip and the collector plate was $15 \mathrm{~cm}$.

\section{S3: Chemical structures of the surfactants.}
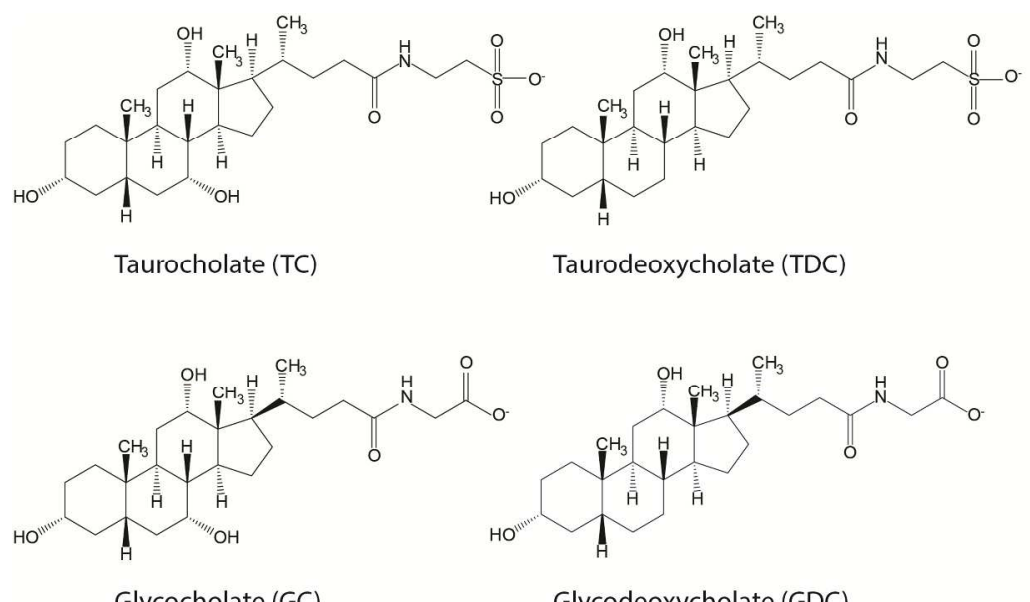

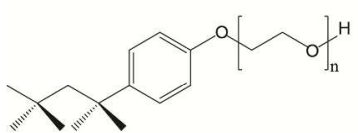

Triton X-100

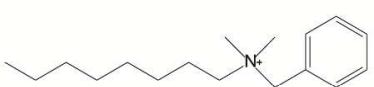

Benzalkonium chloride (BC)

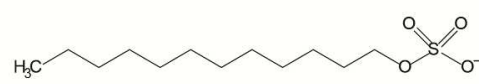

Sodium dodecyl sulfate (SDS)

Figure 1. Chemical structures of the surfactants. 


\section{S4: Stability of FSP-Ins fibers}

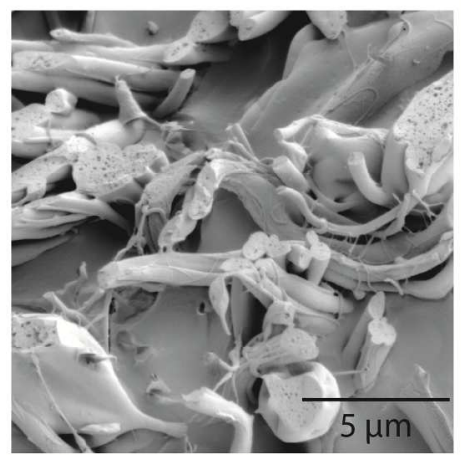

$0 \mathrm{mMTC}$

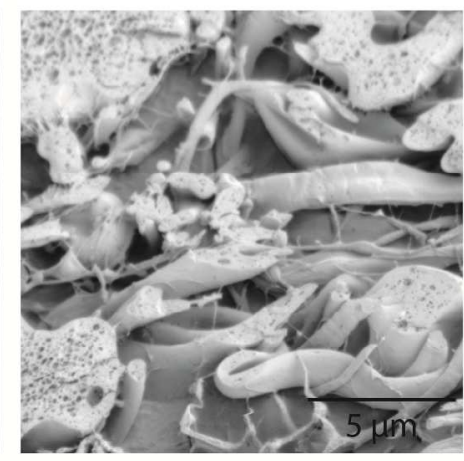

$6 \mathrm{mMTC}$

Figure 2. Structure of FSP-Ins after 2 months of incubation in MES-HBSS without and with TC.

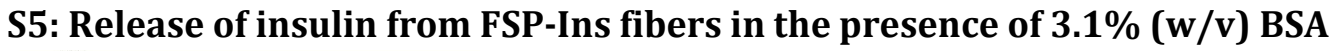

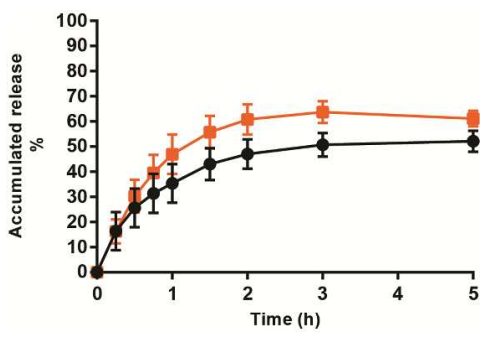

Figure 3. In vitro insulin release from FSP-Ins fibers in MES-HBSS with (orange) and without (black) BSA. Data represent mean \pm SD, $n$ $\geq 3$. 